\title{
Biomedical Informatics and Health Information Technology: a Critical, Pragmatic Collaboration for Clinical Transformation
}

\author{
Kevin B. Johnson, MD, MS ${ }^{1,2}$ and Neal R. Patel, MD, MPH ${ }^{1,2}$ \\ 'Department of Biomedical Informatics, Vanderbilt University Medical Center, Nashville, TN, USA; ${ }^{2}$ Department of Pediatrics, Vanderbilt University \\ Medical Center, Nashville, TN, USA.
}

J Gen Intern Med 36(2):530-2

DOI: $10.1007 / \mathrm{s} 11606-020-05833-y$

(C) Society of General Internal Medicine 2020

$\mathrm{T}$ he widespread adoption of technology and availability of data in biomedicine enable academic health centers (AHCs) to explore new frontiers in care delivery transformation, including precision medicine, augmented intelligence, learning health systems, and EHR optimization. Unfortunately, these technology-driven pursuits are accompanied by evolving threats such as cyberterrorism, changing regulatory frameworks, decreasing revenue, and increasing expenses. ${ }^{1}$ To continue their exploration and address threats, AHCs need to support health information technology (HealthIT) experts, faculty certified in the clinical informatics or trained in biomedical informatics (BMI), and the collaborative and pragmatic partnership between these two groups.

HealthIT and BMI address different AHC needs, with correspondingly different identities and strengths. Typically, BMI represents the "academic" in "AHC"; HealthIT services the "health center operations" part of "AHC." The recent creation of a clinical informatics subspecialty equips the organization with a uniquely trained group of physicians who bridge both cultures.

By providing enterprise-wide, reliable, cost-effective, and cutting-edge technology solutions, HealthIT executes the AHC strategic vision while managing risks. ${ }^{2}$ HealthIT is knowledgeable about software implementation, application development, and infrastructure design and maintenance, combined with software-selection, contract-negotiation, systems-testing/integration, and user-training skills.

In contrast, BMI creates and applies the science of information management - ranging from natural language processing to data modeling to change management - to address vexing problems affecting people, processes, and technology. They uniquely combine their knowledge of biomedicine with how best to manage data, information, knowledge, and people.

Received March 3, 2020

Accepted April 1, 2020

Published online April 22, 2020
They apply their information management methods to areas such as engineering, piloting software products, and evaluating technology/health care workforce change. While their counterparts in HealthIT possess business-oriented skills, and both possess skill in software development, BMI experts possess skill in translating data to knowledge and discovery, critiquing and creating scientific literature, receiving extramural funding, and training the future workforce for both HealthIT and BMI.

In many AHCs, gravitational forces within these two groups - reflecting an existential conflict between the dominance of academics and operations in any given organization - can create friction. Practitioner versus administrative priorities, market disruptors and innovators, health system growth, and policies stifling innovation are all common generators and can lead to attrition and lost productivity. This friction does not have to be unhealthy for the organization. In AHCs, the differing perspectives can be molded into a healthy partnership between HealthIT and BMI, combined with thoughtful and consistent governance. In terms of the former, BMI and HealthIT's unique identities result in different and complementary skills, knowledge, and interests that can create a pragmatic partnership to address issues pervasive in health care. Establishing governance built on this partnership improves prioritization, resource allocation, and even hiring decisions. The presence of this partnership has been recommended by experts in medication safety. ${ }^{3}$

Vanderbilt's leadership spent decades developing the pragmatic partnership between HealthIT and BMI. We attribute our successes below to this partnership, which is achievable in every health system.

Access to longitudinal, research-ready clinical data is essential for precision medicine and predictive analytics/AI. Successful clinical data transformation requires that BMI merge its data-transformation and natural language processing skillset with HealthIT's knowledge about the source of truth for clinical data in the EHR (often not from the EHR itself) and about ways to securely access identified copies of highly sensitive data. As many of these decisions emanate from the "top," both HealthIT and BMI leaders should be accountable for AHC data architecture. An example of this partnership is Vanderbilt's PREDICT, the first national demonstration of 
enterprise-wide use of pharmacogenomics. ${ }^{4}$ We use EHR data to identify and genotype patients likely to require genomeinformed prescribing and then provide therapeutic recommendations during order entry, based on the presence of pharmacologically active genomic variants. In PREDICT, BMI faculty utilized health knowledge about data and natural language processing skills to transform raw data into structured elements from which a prediction model was built. We worked together to operationalize the model and EHR alerts and then collaborated with HealthIT trainers to teach stakeholders how to respond to the alerts. This project was instrumental in the creation of the national All of Us Research Program.

Clinical decision support (CDS) for both $\mathrm{AI}$ and precision medicine is provided through order sets, guidelines, dashboards, alerts, and reminders integrated into care. BMI experts help identify the best approaches; however, these tools require both BMI and HealthIT knowledge to implement them in the EHR and to monitor their performance. These activities required intentional work to evolve a model with subject matter experts to a partnership, as shown in Figure 1. One recent example of CDS partnership occurred during our recent transition to Epic Systems from a locally developed EHR. Our challenge was to transfer over 1000 alerts and reminders to the new system. These alerts were old and unmanaged in many cases. We began by inviting subject matter experts from BMI to work with HealthIT to decide which alerts should be retained and which could be replaced by similar alerts already in Epic. We eventually formed collaborations with business leaders, leading to a true partnership to govern the transition of alerts through a decision support oversight committee co-led by HealthIT and BMI experts. ${ }^{5}$ These efforts are ongoing, as we catalog and monitor all active alerts in our new EHR.

Learning health systems (LHSs) rely on HealthIT/BMI knowledge and expertise to leverage an AHC's vendor-supplied and locally developed infrastructure for comparative effectiveness research. Large or complex innovations often need the scale of an entire health system to demonstrate impact. ${ }^{6}$ Studies like Vanderbilt's SMART trial utilized our LHS, on which both BMI and HealthIT are partners, to design, conduct, and analyze the data. This project began with experts participating in trial design, followed by consultation by our research governance group (BMI and HealthIT) to create a registry for patients combining study-specific data elements from our vendor system, and finally an analytic group powered by LHS members from across the enterprise - many of whom received little to no financial support to engage in this project. Because these groups were already in place, the entire project took less than a year to complete. $^{7}$

A successful partnership requires a non-trivial amount of capital, including funding and protected time to support a cadre of clinical informatics experts to work with HealthIT, a commitment to shared governance to balance HealthIT- versus

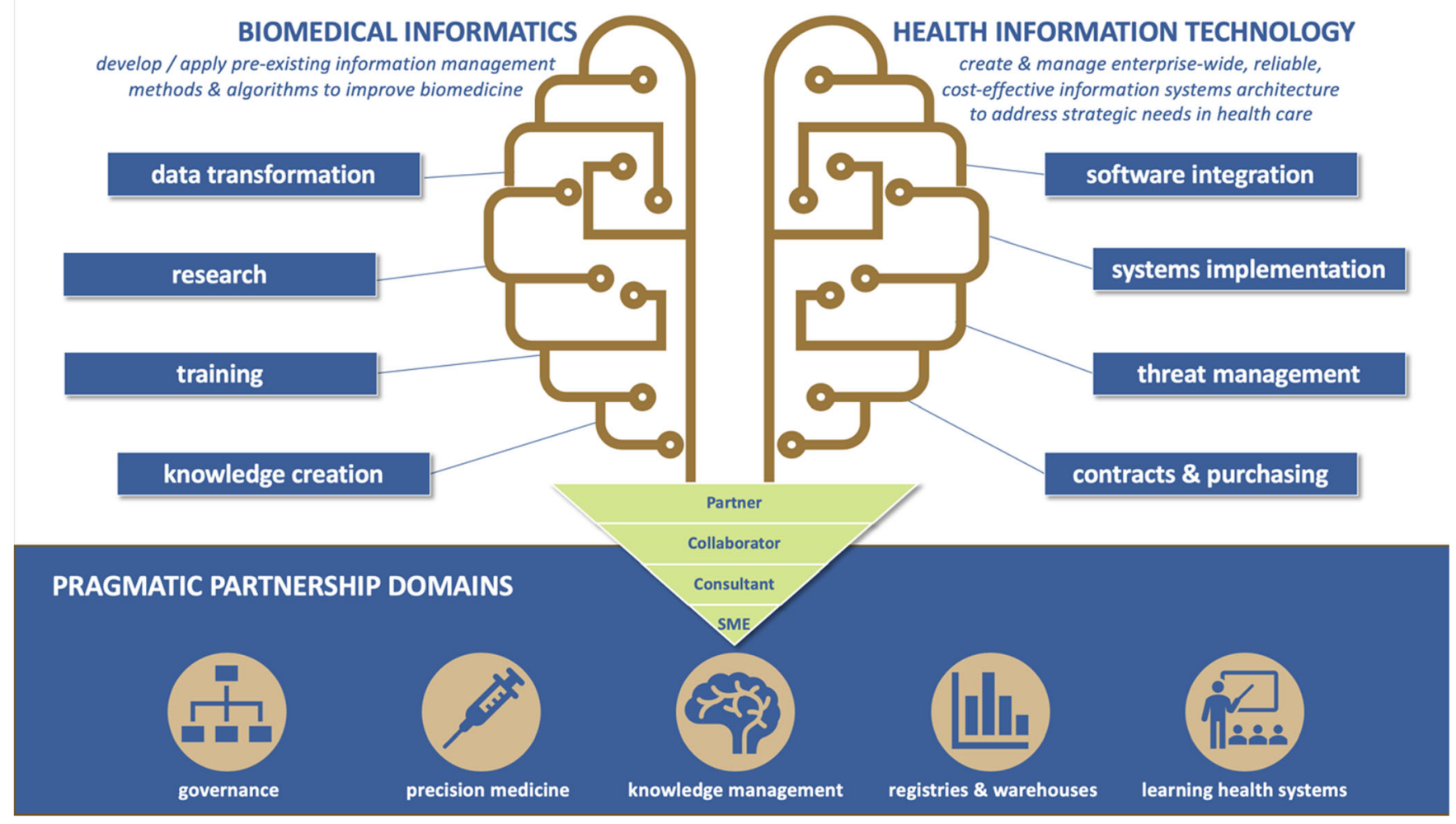

Figure 1 Knowledge and skills comprising a pragmatic partnership between biomedical informatics and health information technology experts. Ideally, knowledge and skills from each organization combine to impact the work in various domains. However, the degree of commitment to the partnership (green triangle) is a key third factor. Subject matter expertise (SME) is a starting point, while significant capital is required to develop respect from each side to be viewed as a consultant, collaborator, and eventually a partner with the other organization. 
BMI- centric tool deployment into the AHC, and intentional efforts to force some degree of collaboration on major initiatives. Note that there are two new sources of expertise who must be jointly recruited by HealthIT and BMI: board-certified physicians who desire to maintain a busy practice but have trained in clinical informatics to work with data and information systems and academically sourced, trained and inclined clinical informaticians who work doing HealthIT, bringing their medical specialty knowledge into the organization.

In summary, AHCs with both biomedical informatics and health information technology expertise are uniquely positioned to leverage the spirit of innovation, empirically driven scientific discovery, and cutting-edge technologic approaches to health care. Both organizations stand to benefit from this pragmatic partnership and, as demonstrated by a few examples at VUMC, may use this relationship to synergistically create a system well-poised for medicine in the information age.

Corresponding Author: Kevin B. Johnson, MD, MS; Department of Biomedical Informatics, Vanderbilt University Medical Center, Nashville, TN, USA? (e-mail: Kevin.johnson@vumc.org).

\section{Compliance with ethical standards:}

Conflict of interest: The authors declare no Conflicts of Interest.

\section{REFERENCES}

1. Balser JR, Stead Ww. Coordinated Management of Academic Health Centers. Trans Am Clin Climatol Assoc. 2017; 128:353-362.

2. Meet the modern healthcare CIO: A business leader that is casting off their traditional IT role. Healthcare IT News. https://www.healthcareitnews.com/ news/meet-modern-healthcare-cio-business-leader-casting-their-traditional-itrole\#: :targetText=As\%20chief\%20information $\% 20$ officers $\% 20$ and,and $\% 20$ scaling\%20the\%20digital\%20business. Accessed 11/26, 2019.

3. Schiff G, Mirica MM, Dhavle AA, Galanter WL, Lambert B, Wright A. A Prescription For Enhancing Electronic Prescribing Safety. Health Aff (Millwood). 2018;37(11):1877-1883.

4. Pulley JM, Denny JC, Peterson JF, et al. Operational implementation of prospective genotyping for personalized medicine: the design of the Vanderbilt PREDICT project. Clin Pharmacol Ther. 2012;92(1):87-95.

5. Wright A, Sittig DF, Ash JS, et al. Governance for clinical decision support: case studies and recommended practices from leading institutions. J Am Med Inform Assoc. 2011;18(2):187-194.

6. Stead WW, Patel NR, Starmer JM. Closing the loop in practice to assure the desired performance. Trans Am Clin Climatol Assoc. 2008;119:185194; discussion 194-185.

7. Semler MW, Self WH, Wanderer JP, et al. Balanced Crystalloids versus Saline in Critically Ill Adults. N Engl J Med. 2018;378(9):829-839.

Publisher's Note Springer Nature remains neutral with regard to jurisdictional claims in published maps and institutional affiliations. 